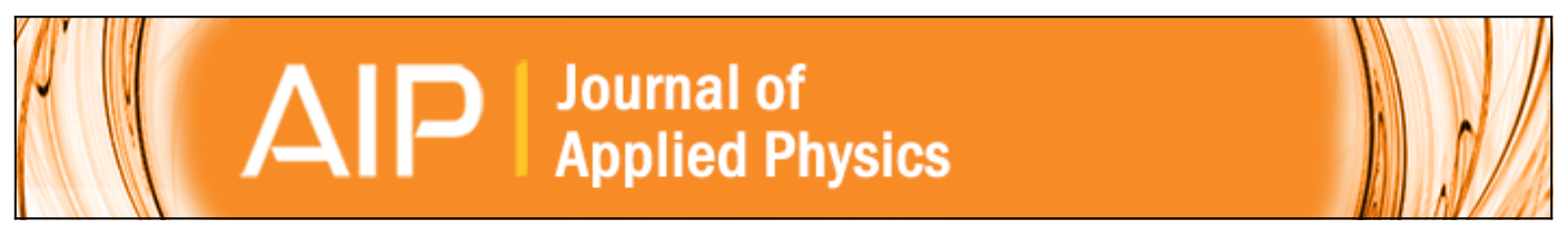

Computation of the expansion parameters of femto-waveguides using a two dimensional -Raman map and guided modes

M. R. Tejerina and G. A. Torchia

Citation: Journal of Applied Physics 114, 153106 (2013); doi: 10.1063/1.4825224

View online: http://dx.doi.org/10.1063/1.4825224

View Table of Contents: http://scitation.aip.org/content/aip/journal/jap/114/15?ver=pdfcov

Published by the AIP Publishing

AlP Re-register for Table of Content Alerts 


\title{
Computation of the expansion parameters of femto-waveguides using a two dimensional $\mu$-Raman map and guided modes
}

\author{
M. R. Tejerina and G. A. Torchia ${ }^{\text {a) }}$ \\ Centro de Investigaciones Ópticas CONICET La Plata CIC Camino Centenario y $506 \mathrm{~s} / \mathrm{n}$ M.B. Gonnet (1897) \\ Pcia Buenos Aires, Argentina
}

(Received 5 July 2013; accepted 30 September 2013; published online 17 October 2013)

\begin{abstract}
The expansion parameters of a numerical elastic model that simulates femtosecond laser-material interactions are computed. To achieve this, two dimensional micro-Raman mapping, an elasticoptic finite element model, and the experimental intensity profile of the guided modes were used. To complement this data, the Raman deformation potential constants of the lithium niobate $\mathrm{A}_{1}\left(\mathrm{TO}_{4}\right)$ phonon were obtained and compared with those reported in previous works. (C) 2013 AIP Publishing LLC. [http://dx.doi.org/10.1063/1.4825224]
\end{abstract}

\section{INTRODUCTION}

Since ultra-fast laser writing (pulses of hundreds of femtoseconds) emerged, it has become a feasible tool for "onestep" optical circuit fabrication. An important advantage of this fabrication method is that it allows the processing of an unlimited number of optical materials. ${ }^{1}$ Nonlinear laser absorption in a transparent material is the key process for waveguide fabrication. This occurs because of the incidence of many infrared photons in the tightly focused region. Then, a micro-explosion and re-solidification increase the refractive index in the nearby region of the focused volume. So, by translating the focus through the bulk transparent material, an optical waveguide can be formed. ${ }^{1}$

$\mathrm{LiNbO}_{3}$ crystal is an optical material that is widely used in many commercial photonic devices due to its excellent electro-optical, piezo-optical, and acousto-optical properties. $^{2}$ Optical circuits made using different techniques in this material are commonly used in commercial devices for optical communications and in other technological fields. ${ }^{3}$

When the femtosecond laser writing waveguide fabrication method emerged, there was not a quantitative description of the mechanical properties resulting from the nonlinear interaction, particularly for $\mathrm{LiNbO}_{3}$ crystals. For this reason, much research has been carried out into forming waveguides with different processing parameters (velocity, pulse duration, and pulse energy), and testing the characteristics of the guided radiation. ${ }^{4,5}$ As a result, a large number of successful integrated guiding structures have been reported. ${ }^{6-10}$

Following this, knowledge about femtosecond laser written waveguides (herein "femto-waveguides") in $\mathrm{LiNbO}_{3}$ has been obtained via experimental studies and a numerical model approach. ${ }^{11,12}$ This elastic model has played an important role, particularly in the study of the origin of femto-waveguides. The model consists of an ellipse that statically expands within an anisotropic domain, and the mechanical deformation surrounding the ellipse generates a refractive index increment, which allows waveguiding. Following this initial model, ${ }^{11,12}$ various studies aimed to investigate the residual stress in femto-waveguides using

a)gustavot@ciop.unlp.edu.ar numerical models. ${ }^{13-15}$ Another study retrieved characteristics of the material structure and made a qualitative residual strain analysis in femto-waveguides using micro-Raman measurements. ${ }^{14}$ Subsequently, ${ }^{15}$ a femto-waveguide was analyzed using the following resources: $\mu$-Raman analysis in a linear scanning path through the waveguide; linear deformation potential theory; and the elastic finite element model. Despite these significant advances, more information is still required to optimize the design of femto-waveguides in $\mathrm{LiNbO}_{3}$.

To achieve this aim, in this report, a bi-dimensional analysis is performed using the following strategy. First, a bi-dimensional $\mu$-Raman mapping of femto-waveguides in $\mathrm{Nd}: \mathrm{Mg}: \mathrm{LiNbO}_{3}$ is fitted with an elastic model to determine the optimized expansion parameter rates. Second, using the hydrostatic pressure Raman constant $(d w / d p)$, the values of the expansion parameters are estimated for the relevant cases. Finally, to unambiguously determine which expansion parameters are appropriate; the optical guided modes are experimentally measured and compared with the numerically determined ones.

\section{MATERIALS AND METHODS}

\section{A. Waveguide fabrication}

In this work, the analyzed waveguide was fabricated in a $\mathrm{Nd}: \mathrm{Mg}: \mathrm{LiNbO}_{3} \mathrm{z}$-cut crystal sample. The ratios of $\mathrm{Nd}$ - and $\mathrm{Mg}$-doping were $0.3 \%$ and $5 \%$, respectively. The refractive index modification that allows optical waveguiding to occur was generated with a $796 \mathrm{~nm}$ Ti:sapphire ultrafast laser. The duration of the pulses was $120 \mathrm{fs}$, with a repetition rate of $1 \mathrm{kHz}$, and pulse energy of $3 \mu \mathrm{J}$. A $20 \times(\mathrm{NA}=0.4)$ microscope objective was used to focus the laser beam inside the crystal. A displacement velocity of $25 \mu \mathrm{m} / \mathrm{s}$ along the $y$-direction (normal to the propagation direction) was set. The structure modification performed within the crystal allowed guidance of radiation. ${ }^{16}$

\section{B. Bi-dimensional $\mu$-Raman mapping}

The femto-waveguides were studied using back-scattering $\mu$-Raman spectroscopy mapping in the $\mathrm{y}(\mathrm{zz}) \mathrm{y}$ configuration. 
For this experiment, a $200 \mathrm{~mW}$ argon ion laser centered at $488 \mathrm{~nm}$ and a Confocal Olympus BX-41 employing a $50 \times$ $(\mathrm{NA}=0.25)$ microscope objective were used. A set of notch filters and a polarizer were also used in this experiment. ${ }^{16}$

A 2D map of the waveguides was generated with $\mathrm{x}$ - and z-direction steps of $1 \mu \mathrm{m}$. The Raman spectrum for the $\mathrm{LiNbO}_{3} \mathrm{y}(\mathrm{zz}) \mathrm{y}^{16}$ configuration was measured and stored at each point of the map. Then, fitting the $\mathrm{A}_{1}(\mathrm{TO})_{4}$ phonon with a Lorentzian function, the energy shift of the phonon in the mapped zone was obtained as a 3D surface (wavenumber variation of phonon vs. $z$-displacement vs. $\mathrm{x}$-displacement). Subsequently, as a non-symmetric model is not available in the literature, the four quadrants shown in Figure 1(a) were averaged. The average Raman shift field is used to perform the fitting in this work and is detailed in Figure 1(b).

\section{Mode coupling}

To perform light coupling to the femto-waveguide, the well-known "end-fire" method ${ }^{6}$ was implemented. Using this technique, the spatial distribution of the guided mode intensity at the output was measured. This experiment was carried out using a $532 \mathrm{~nm}$ continuous wave (cw) solid state laser, two $20 \times$ microscopic objectives (for input and output coupling), a micro-positioning system, and a standard beam profile analyzer from Newport Corporation.

\section{Elastic and optical finite element model}

\section{Elastic finite element model}

Many complex phenomena occur in the femtosecond laser interaction, e.g., material ionization, expansion shock wave, and direct affected material re-solidification. The most of them are not quantitatively described. So, the result of the whole process from a point of view of the surrounding residual strain field was modeled in literature, ${ }^{11,15,17}$ as a pseudoarbitrary mechanical expansion of an ellipse in an "infinite" medium. Consequently, the expansion and geometrical parameters of this model represent, in a first order of

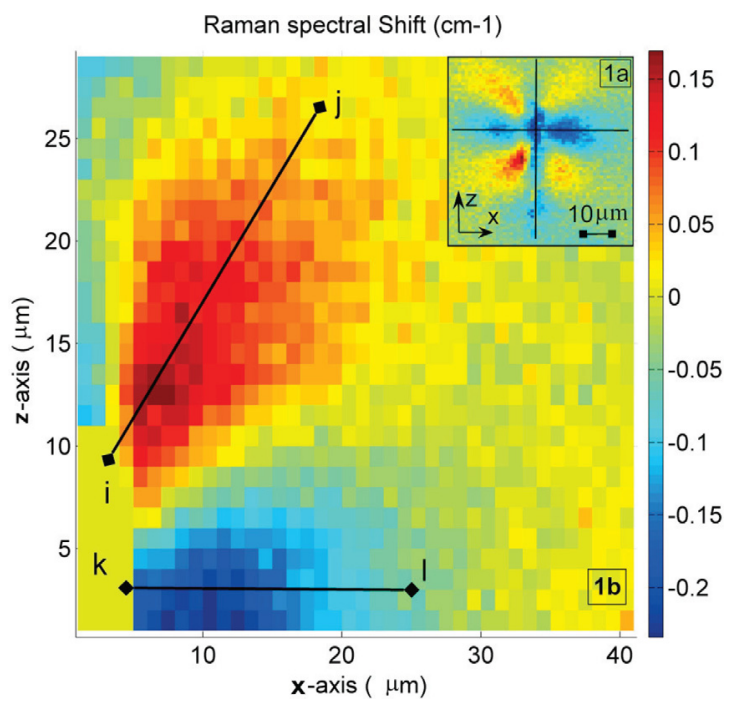

FIG. 1. (a): 2D Raman spectral shift of the waveguide zone; (b) averaged Raman spectral shift used in this work. approximation, the sum of all the mentioned phenomena. Anyway, the ellipse geometry parameters of the model (a and b) can be related with the region of the material, which is exposed to an energy higher than the modification threshold (material fluency). Also, the ellipse expansion parameters of the model $(\alpha$ and $\beta)$ can be related with a density change of the direct-affected zone. ${ }^{11}$

As mentioned, a numerical model can be used to estimate the residual strain field, and, thus, the refractive index field in femto-waveguides. In the current work, the following general assumptions, validated in a previous work, ${ }^{17}$ are implemented. These are elastic material, static expansion, plane strain deformations $\left(\varepsilon_{y y}=0\right)$, and an orthotropic approximation for the $\mathrm{LiNbO}_{3}$ compliance matrix.

In particular, in the numerical model used in this work, a quarter of an ellipse (with initial horizontal and vertical radii $a$ and $b$, respectively) expands with the expansion parameters $\alpha(\Delta a / a)$ and $\beta(\Delta b / b)$. A scheme of the system, with geometrical parameters detailed, is shown in Figure 2.

To compute the refractive index field in the z-direction $\left(n_{\mathrm{z}}\right)$ due to the piezo-optic effect, a code reported in a previous work was used. ${ }^{15}$

\section{Guided modes using the finite element model}

As the femto-waveguides generated in z-cut lithium niobate have a refractive index increment in only the z-direction, ${ }^{12}$ the scalar Helmholtz equation can be used to estimate the guided modes in the computed refractive index field. ${ }^{18}$ To solve this differential equation, the finite element method was used. This method can be used to compute guided modes for arbitrarily shaped refractive index fields. ${ }^{19}$

To simulate the intensity of guided modes in the numerical model, a MATLAB code was developed following the steps indicated by Okamoto et al.. ${ }^{19}$ The refractive index field used for this purpose was extracted from the numerical model mentioned in Sec. II D 1.

The mesh implemented in the optical model was generated using the "Distmesh" code. ${ }^{20}$ In this mesh, the elements

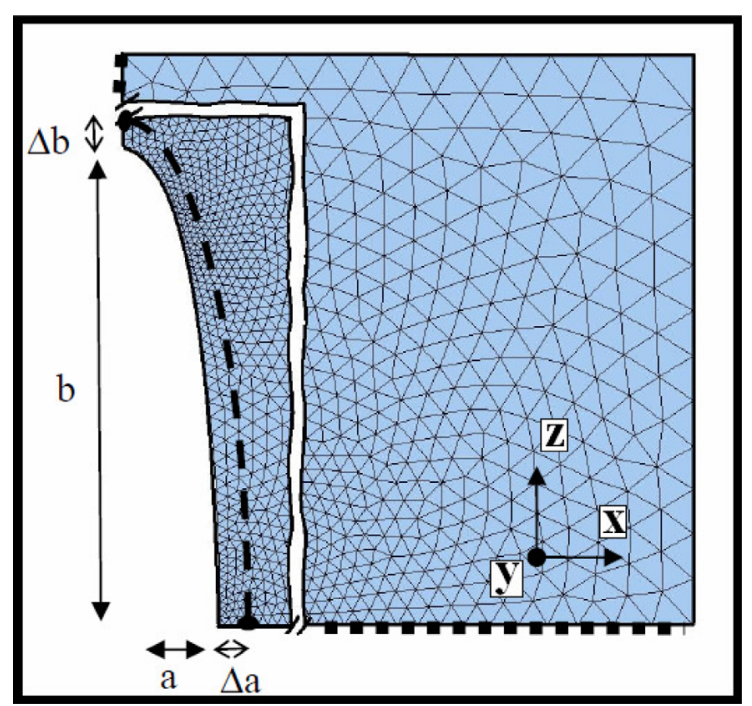

FIG. 2. Expansion parameters and geometry of the linear elements used in the elastic model presented in this work. 
used to discretize the domain were linear triangular elements, as sketched in Figure 2.

\section{THEORY AND CALCULATION METHOD}

\section{A. Raman linear potential deformation theory}

To relate the deformation field of the lattice to the spectral shifting of the optical phonons, Raman deformation potential theory (RDPT) was implemented. Using this theory, and taking into account crystallographic properties of $\mathrm{LiNbO}_{3}$ and the plane strain assumption $\left(\varepsilon_{\mathrm{yy}}=0\right)$, Eq. (1) can be obtained for $\mathrm{A}_{1}$ phonons ${ }^{17}$

$$
\Delta w(z, x, \alpha, \beta)=e \cdot \varepsilon_{\mathrm{xx}}(z, x, \alpha, \beta)+f \cdot \varepsilon_{\mathrm{zz}}(z, x, \alpha, \beta),
$$

where $\Delta w$ is the spectral shift of a phonon of symmetry $\mathrm{A}_{1}$ (transverse optical); $e$ and $f$ are the $\mathrm{A}_{1}$ phonon deformation potential constants; $\varepsilon_{x x}$ and $\varepsilon_{z z}$ are the elements of deformation tensor; $z$ and $x$ are the spatial coordinates; and $\alpha$ and $\beta$ are the expansion coefficients.

In the particular case where a hydrostatic pressure is applied to a solid, Eq. (2) is obtained ${ }^{17}$

$$
d w d p_{i}=2 \cdot C_{1} \cdot e_{i}+C_{2} \cdot f_{i}
$$

where $C_{1}$ and $C_{2}$ are constants that depend only on the material compliance matrix; ${ }^{17} e_{i}$ and $f_{i}$ are the phonon deformation potential constants of phonon $i$; and $d w / d p$ is the variation of linear frequency with hydrostatic pressure of phonon $i$.

\section{B. Computation of optimal expansion parameters}

In a previous work, ${ }^{21}$ the geometrical parameters $a=1.7 \mu \mathrm{m}$ and $b=8.5 \mu \mathrm{m}$ were computed as optimal values for this type of waveguide. These values were used in the current numerical approach to estimate the expansion parameters. To reach this aim, the steps outlined below were followed:

(1) The optimal $\beta / \alpha$ rates of the $2 \mathrm{D}$ Raman fitting were determined.

(2) Then, the values of the geometrical parameters $(\beta$ or $\alpha)$ for the relevant cases were computed using the Raman spectral shift of the $A_{1}\left(\mathrm{TO}_{4}\right)$ phonon under hydrostatic pressure.

(3) Finally, the theoretical guided modes for the numerically allowed cases (those that match steps 1 and 2) were compared with the measured guided mode intensity from the femto-waveguide.

Below, a more detailed description of steps 1 and 2 is presented. A more detailed description of step (3) is presented in Sec. IV.

Step 1: Optimal $\beta / \alpha$ rates for Raman map fitting.

Using the $\mu$-Raman mapping described in Sec. III A, and the elastic finite element model described in Sec. II D 1, the quadratic error was computed for a broad range of expansion parameter rates ( $\beta / \alpha)$ using Eq. (3). The $\beta / \alpha$ values that minimize Eq. (3) were set as optimal

$$
\operatorname{TSE}(\beta / \alpha)=\sum_{i=1}^{n}\left(\Delta A T o_{\exp }\left(z_{i}, x_{i}\right)-\Delta A T o_{\text {num }}\left(z_{i}, x_{i}, \beta / \alpha\right)\right)^{2} .
$$

Step 2: Computation of the expansion parameters $(\alpha$ and $\beta$ ) using the previously reported value of hydrostatic pressure Raman shift $\left(d w / d p_{\text {exp }}\right)$.

As mentioned above, for the different cases considered to be optimal in step 1 , the values of $\alpha$ and $\beta$ that fit the known hydrostatic pressure Raman shift value $\left(d w / d p_{\text {exp }}\right)$ were determined in this second step.

The $\beta / \alpha$ rates that were computed to be optimal in step 1 (more information about this procedure will be explained in Sec. IV), are the following:

case (i): $\beta / \alpha>10^{3}$

case (ii): $\beta / \alpha<10^{-3}$

(i) In this case, for simplicity, we set $\alpha=0$. So, using this condition and substituting Eq. (1) into Eq. (3), Eq. (4) is obtained

$$
\begin{aligned}
\operatorname{TSE}(\alpha)= & \sum_{i=1}^{n}\left(\Delta A T o_{\exp }\left(z_{i}, x_{i}\right)\right. \\
& \left.-\left[e \cdot \varepsilon_{\mathrm{Xx}}\left(z_{i}, x_{i}, \beta\right)+f \cdot \varepsilon_{\mathrm{Zz}}\left(z_{i}, x_{i}, \beta\right)\right]\right)^{2} .
\end{aligned}
$$

By minimizing Eq. (4), the optimal values of $e$ and $f$ were calculated for different values of $\beta$. Then, by inserting this value into Eq. (2), the numerical $d w / d p$ value $\left(d w / d p_{\text {nит }}\right)$ as a function of $\beta$ was obtained.The optimal value of $\beta\left(\beta_{o}\right)$ is that which satisfies Eq. (5).

$$
d w / d p_{\text {num }}\left(\beta_{0}\right)=d w d p_{\exp },
$$

where $d w / d p_{\text {exp }}$ is a known constant for the $\mathrm{A}_{1}(\mathrm{TO})_{4}$ phonon.

(ii) In this case, we set $\beta=0$ for simplicity. So, using this condition and substituting Eq. (1) into Eq. (3), we obtain Eq. (6)

$$
T S E=\sum_{i=1}^{n}\left(\Delta A T o_{\exp }\left(z_{i}, x_{i}\right)-\left[e \cdot \varepsilon_{\mathrm{xx}}\left(z_{i}, x_{i}, \alpha\right)+f \cdot \varepsilon_{\mathrm{zz}}\left(z_{i}, x_{i}, \alpha\right)\right]\right)^{2} .
$$

Analogous to the previous case, the numerical value of $d w / d p\left(d w / d p_{\text {num }}\right)$ can be computed using Eq. (2), as a function of $\alpha$. So, the optimal value of $\alpha, \alpha_{o}$, will be that which satisfies Eq. (7)

$$
d w / d p_{\text {num }}\left(\alpha_{0}\right)=d w / d p_{\exp } .
$$

It is important to remark that, in each case ( $i$ and $i i)$, there is a different expansion parameter rate $(\beta / \alpha)$ that minimizes the total square error (TSE) of the bi-dimensional Raman shift fitting. So, the optimal values of $\alpha$ and $\beta$ were obtained using the hydrostatic Raman behavior for both cases. Subsequently, using these values, two different refractive index fields can be obtained numerically. 

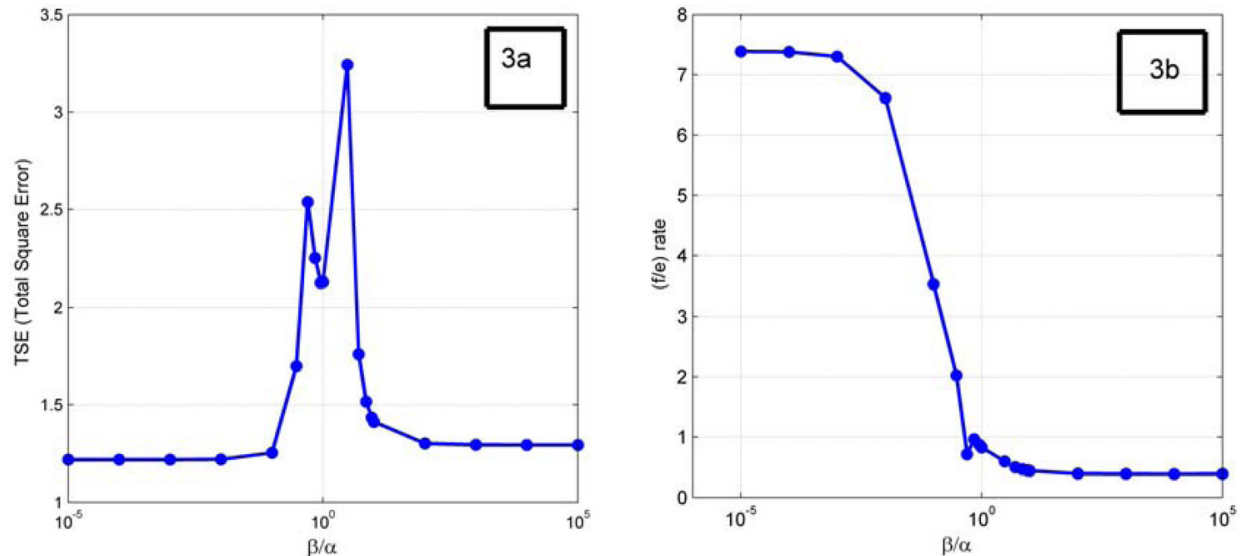

FIG. 3. (a) The TSE against $\beta / \alpha$. (b) $\mathrm{f} / \mathrm{e}$ versus $\beta / \alpha$.
To confirm which of the mentioned cases ( $i$ or $i i)$ corresponds to the waveguide structures studied in this work, a numerical computation of the guided optical modes for both numerical strain fields was performed and compared to the experimental data.

\section{RESULTS AND DISCUSSION}

\section{A. Total summation error and $f / e$ rates for different $\beta / \alpha$ expansion parameter rates}

To understand the behavior of the TSE as a function of $\beta / \alpha$, the data obtained were plotted and are shown in Figure 3(a). It can be seen that TSE is minimized in the following cases: (i) when $\beta / \alpha<10^{-1}$ (left branch), and (ii) when $\beta / \alpha>10^{1}$ (right branch). Each branch has an absolute minimum value of TSE. This fact indicates that, a priori, both branches are of interest in the current approach.

In Figure 3(b), where the fle rate vs. $\beta / \alpha$ is plotted on the logarithmic scale, it can be seen that the fle relationship is almost constant along the left and right branches $(\alpha \ll \beta$ and $\beta \ll \alpha$, respectively). This fact indicates that, in each branch, the $\varepsilon_{\mathrm{xx}}(\mathrm{z}$, $\mathrm{x})$ and $\varepsilon_{\mathrm{zz}}(\mathrm{z}, \mathrm{x})$ solutions for different $\beta / \alpha$ are proportional. This means that, for example: $\varepsilon_{\mathrm{xx}} / \varepsilon_{\mathrm{zz}}\left(\beta / \alpha=10^{-4}\right)=\varepsilon_{\mathrm{xx}} / \varepsilon_{\mathrm{zz}}$ $\left(\beta / \alpha=10^{-5}\right)$; and $\left.\varepsilon_{\mathrm{xx}} \varepsilon_{\mathrm{zz}}\left(\beta / \alpha=10^{4}\right)\right)=\varepsilon_{\mathrm{xx}} / \varepsilon_{\mathrm{zz}}\left(\beta / \alpha=10^{5}\right)$.

In cases (i) and (ii), as stated in Sec. III B, $\beta=0$ was set for the first case and $\alpha=0$ for the second.

\section{B. Numerical Raman hydrostatic constant vs. expansion parameters}

To find the optimal $\beta$ for case (i) and the optimal $\alpha$ for case (ii), the Raman hydrostatic pressure constant $d w / d p$ definition was used. According to Eq. (2), $d w / d p$ can be expressed as a linear function of $e, f$, and the known compliance matrix elements. ${ }^{17}$

In Figures 4(a) and 4(b), using Eq. (2), $d w / d p_{\text {num }}$ was plotted as a function of $\beta(\alpha=0)$ for case (i); and as a function of $\alpha(\beta=0)$ for case (ii). In both cases, taking into account the value of $d w / d p_{\text {exp }}$ experimentally determined in the literature $\left(d w / d p_{\exp }=0.15\right),{ }^{22}$ the values of $\alpha$ or $\beta$ were obtained. This was carried out by fitting the computed points with a rational function (solid line in Figures 4(a) and 4(b)). The expansion parameters gave $\beta=0.015(\alpha=0)$ for case (i), and $\alpha=0.02(\beta=0)$ for case (ii).

In summary, we found two cases ( $i$ and $i i$ ) which fulfill the constraints required by the minimum TSE (minimizing Eq. (3)) and the experimental Raman hydrostatic pressure behavior for phonon $\mathrm{A}_{1}\left(\mathrm{TO}_{4}\right)$ (Eqs. (5) and (7)). These cases are:

(i) $\alpha=0, \beta=0.015$,

(ii) $\alpha=0.02, \beta=0$.

\section{Optical mode comparison}

Cases (i) and (ii) are allowed by the numerical 2D $\mu$-Raman fitting and the Raman hydrostatic pressure behavior condition (Eqs. (6) and (7)). To define which of these two cases corresponds to the refractive index field of the femtowaveguide, the characteristics of the guided modes were analyzed. This was performed using the numerical model described in Sec. IID 2 and the experimental data measured for the guided mode (Sec. II C).
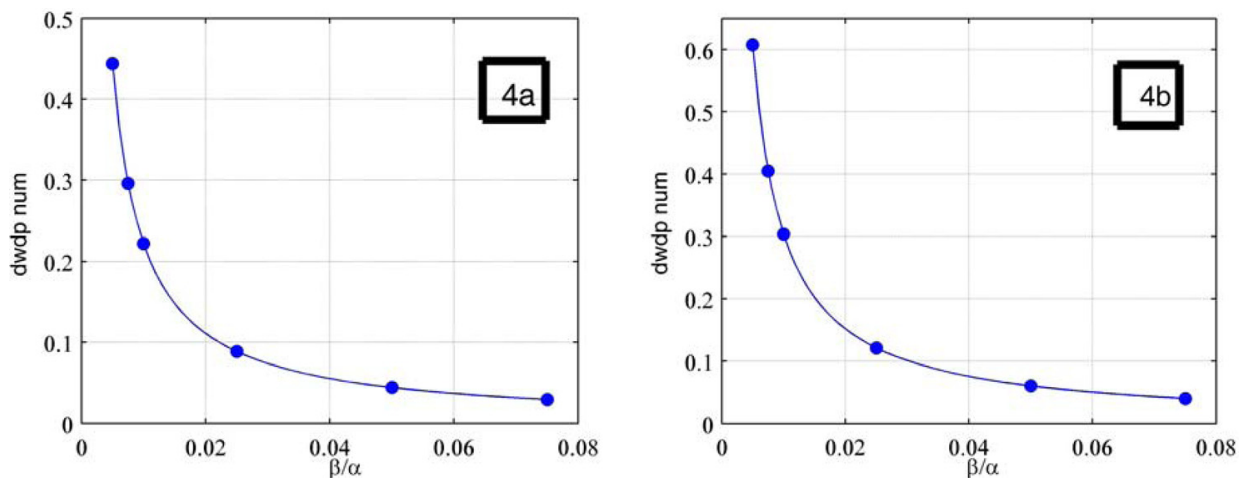

FIG. 4. (a) $d w / d p$ vs. $\beta_{\alpha=0}$. (b) $d w / d p$ vs. $\alpha_{\beta=0}$. 


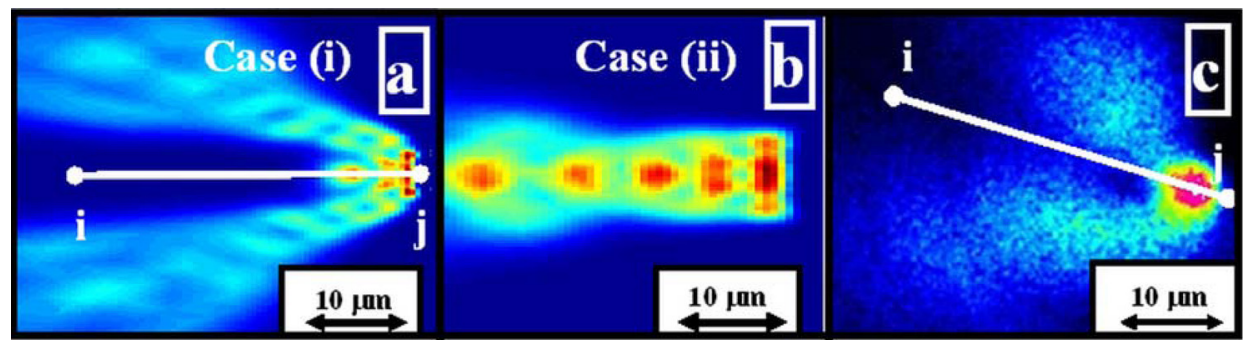

FIG. 5. Guided mode intensity in: (a) case (i), (b) case (ii), and (c) the experimental.
In Figures 5(a)-5(c), the guided mode intensity corresponding to the simulated cases (i) and (ii), and the experimental guided mode intensity are shown, respectively. It can be observed that the numerical guided mode depicted in Figure 5(a) (case (i)) correlates well with the experimental guided mode (Figure 5(c)). It can also be observed, by comparing Figures 5(b) and 5(c), that case (ii) does not agree with the measured guided mode intensity, so it does not correspond to the analyzed waveguide.

The relation $\beta \gg \alpha$ obtained in this work agrees with the expansion parameters relation $(\beta / \alpha)$ obtained using other expansion models in a previous report. ${ }^{17}$ In that report, the computed horizontal expansion parameters $\left(\alpha_{\mathrm{i}}\right)$ are negligible, as in the present case. Similarly, the obtained fle value computed using the current model $(\mathrm{f} / \mathrm{e} \approx 0.45)$ is close to that obtained in the previous report $(f / e \approx 0.6)$.

The values of $e$ and $f$ obtained from the current model are around -190 and -90 , respectively; in a previous report, ${ }^{17}$ they give values of -160 and -100 , respectively. The difference between the deformation potential constants obtained in these works is at most $15 \%$. So, in general terms, good accordance is reached between both works, although different expansion models and strategies were used to obtain the values.

However, there is another recent report ${ }^{23}$ in which $e=-450$ and $f=-195$ were determined, by simultaneously using different resources. In this report, the fle rate was 0.43 , which is close to the value determined in our work $(f / e=0.45)$. However, the $e$ and $f$ values do not concur with our results.

We think that the main cause of this disagreement is as follows: in our work, the value of $d w / d p=0.15$, experimentally measured by Filho et al., ${ }^{22}$ was used, and, a $d w / d p=0.33$ can be inferred by substituting $e$ and $f$ values, obtained by Pezzotti et al., ${ }^{23}$ into Eq. (2). This generates the difference observed between the values of $e$ and $f$ in both works.

Finally, another interesting observation, from Figure $5(\mathrm{c})$, is that the guided mode has a particular geometric form, which can be called a "horseshoe," and its axis of symmetry (ij-axis) is not aligned with the crystal $\mathrm{x}$-axis. In the numerical model, shown in Figure 5(a), the same geometry can be observed, but its symmetry axis ( $i j$-axis) is aligned with the crystal $\mathrm{x}$-axis. We think that this difference is due to the non-symmetric expansion with respect to the crystal $\mathrm{x}$-axis that seems to occur in the analyzed laser-matter interaction.

The asymmetric feature of the experimental propagated modes may be mainly related with an asymmetric focal volume generated by the non-linear propagation of the femtosecond pulses. $^{24}$ This fact can generate an asymmetric expansion respect to the $\mathrm{x}$-axis, and thus, a refractive index field with the same feature. A non-symmetrical model is required to enable exhaustive analysis of this behavior in future work.

\section{CONCLUSION AND FUTURE WORK}

A femto-waveguide written in $\mathrm{Nd}: \mathrm{Mg}: \mathrm{LiNbO}_{3}$ was analyzed using theoretical, numerical, and experimental resources. The resources were: Raman deformation potential theory, an elastic-optic finite element model, bi-dimensional Raman mapping, an experimental measurement of the guided mode intensity, and observation of the Raman shift under hydrostatic pressure.

The main target of this work was to compute the expansion parameters of the elastic model that simulates femtosecond-material interaction, as well as to obtain the values of the deformation potential constants.

The simulated guided intensity, for appropriate expansion parameters, showed good accordance with the experimental measurement. Moreover, the computed expansion parameter rates were in good accordance with a previous report involving femto-waveguides, although a different strategy was implemented to obtain the values. In both works, a dominance of the vertical displacement (x-direction) of the expanded area was observed. In addition, the Raman deformation potential constants were, at most, $15 \%$ different between previous reports and the current work involving femto-waveguides.

This work confirms the need to expand research into the femtosecond laser-matter interaction, as well as the necessity to develop non-symmetric numerical models to characterize the femto-waveguides in more depth. We believe this step should be undertaken in future work.

In addition, an interesting "incompatibility" between the Raman behavior under hydrostatic and non-hydrostatic strain has been experimentally measured and reported in other works. Considering that $\mu$-Raman mapping is an important tool for three-dimensional strain field computing, it is necessary improving this approach to solve the mentioned discrepancies.

\section{ACKNOWLEDGMENTS}

This work was partially supported by the Agencia de Promoción Científica y Tecnológica under project PICT2010-2575, and by the CONICET under project PIP 5934.

${ }^{1}$ K. M. Davis, K. Miura, N. Sugimoto, and K. Hirao, Opt. Lett. 21(21), 1729 (1996).

${ }^{2}$ Y. Kusminov, Lithium Niobate Crystals (Cambridge International Science Publishing, Cambridge, 1997). 
${ }^{3}$ G. Lifante, Integrated Photonics: Fundamentals (John Wiley \& Sons, London, 2003).

${ }^{4}$ C. Schaffer, Ph.D. dissertation, Harvard University, 2001.

${ }^{5} \mathrm{H}$. Misawa and S. Juodkazis, 3D Laser Microfabrication: Principles and Applications (Wiley-VCH, Weinheim, 2006).

${ }^{6}$ R. Gattas, Ph.D. dissertation, Harvard University, 2006.

${ }^{7}$ W. Yang, Ph.D. dissertation, University of Southampton, 2008.

${ }^{8}$ B. Poumellec, L. Sudrie, M. Franco, B. Prade, and A. Mysyrowicz, Opt. Express 11, 1070 (2003).

${ }^{9}$ M. Richardson, A. Zoubir, C. Rivero, C. Lopez, L. Petit, and K. Richardson, Proc. SPIE 5347, 18 (2004).

${ }^{10}$ R. Ramponi, R. Osellame, and G. Cerullo, "Femtosecond laser micromachining photonic and microfluidic devices in transparent materials," in Photonic and Microfluidic Devices in Transparent Materials Series, Vol. 123 Topics in Applied Physics (Springer, 2012).

${ }^{11}$ J. Burghoff, S. Nolte, and A. Tunnermann, Appl. Phys. A 89, 127 (2007).

${ }^{12}$ J. Burghoff, C. Grebing, S. Nolte, and A. Tunnermann, Appl. Surf. Sci. 253, 7899 (2007).

${ }^{13}$ S. Nolte, J. Burghoff, M. Will, and A. Tunnermann, Proc. SPIE 5340, 164 (2004).
${ }^{14}$ M. Will, J. Burghoff, S. Nolte, and A. Tunnermann, Proc. SPIE 5714, 261 (2005).

${ }^{15}$ M. Tejerina and G. A. Torchia, Appl. Phys. A 110, 591 (2013).

${ }^{16}$ A. Ródenas, L. M. Maestro, M. O. Ramirez, G. A. Torchia, L. Rosso, F. Chen, and D. Jaque, J. Appl. Phys. 106, 013110 (2009).

${ }^{17}$ M. R. Tejerina, D. Jaque, and G. A. Torchia, J. Appl. Phys. 112, 123108 (2012).

${ }^{18}$ B. M. A. Rahman, Prog. Electromagn. Res. 10, 187 (1995).

${ }^{19} \mathrm{~K}$. Okamoto, Fundamentals of Optical Waveguides (Elsevier, USA, 2006).

${ }^{20}$ P. O. Persson and G. Strang, SIAM Rev. 46(2), 329 (2004).

${ }^{21}$ M. R. Tejerina, D. Jaque, and G. A. Torchia, "A 2D $\mu$-Raman correlation of femtosecond written waveguides using an elastic model in Lithium Niobate," Opt. Materials (under review).

${ }^{22}$ M. Filho, V. Lemos, and F. Cerdeira, J. Raman Spectrosc. 15, 367 (1984).

${ }^{23}$ G. Pezzotti, H. Hagihara, and W. Zhu, J. Phys. D: Appl. Phys. 46, 145103 (2013).

${ }^{24}$ A. Ferrer, A. R. de la Cruz, D. Puerto, W. Gawelda, J. A.Vallés, M. A. Rebolledo, V. Berdejo, J. Siegel, and J. Solis, J. Opt. Soc. Am. B 27, 1688 (2010). 